\title{
Identification of Modules Related to Programmed Cell Death in CHD Based on EHEN
}

\author{
Xu Jia, ${ }^{1}$ Wan Li, ${ }^{1}$ Zhengqiang Miao, ${ }^{1}$ Chenchen Feng, ${ }^{1}$ Zhe Liu, ${ }^{1}$ Yuehan He, ${ }^{1}$ Junjie Lv, ${ }^{1}$ \\ Youwen Du, ${ }^{1}$ Min Hou, ${ }^{1}$ Weiming He, ${ }^{2}$ Danbin $\mathrm{Li}^{3}{ }^{3}$ and Lina Chen ${ }^{1}$ \\ ${ }^{1}$ College of Bioinformatics Science and Technology, Harbin Medical University, Harbin, Heilongjiang 150000, China \\ ${ }^{2}$ Institute of Opto-Electronics, Harbin Institute of Technology, Harbin, Heilongjiang 150000, China \\ ${ }^{3}$ Surgery, The First Affiliated Hospital of Harbin Medical University, Harbin, Heilongjiang 150000, China \\ Correspondence should be addressed to Weiming He; hewm@hit.edu.cn and Lina Chen; chenlina@ems.hrbmu.edu.cn
}

Received 27 March 2014; Accepted 28 May 2014; Published 15 July 2014

Academic Editor: Dong Wang

Copyright (C) $2014 \mathrm{Xu}$ Jia et al. This is an open access article distributed under the Creative Commons Attribution License, which permits unrestricted use, distribution, and reproduction in any medium, provided the original work is properly cited.

The formation and death of macrophages and foam cells are one of the major factors that cause coronary heart disease (CHD). In our study, based on the Edinburgh Human Metabolic Network (EHMN) metabolic network, we built an enzyme network which was constructed by enzymes (nodes) and reactions (edges) called the Edinburgh Human Enzyme Network (EHEN). By integrating the subcellular location information for the reactions and refining the protein-reaction relationships based on the location information, we proposed a computational approach to select modules related to programmed cell death. The identified module was in the EHEN-mitochondria (EHEN-M) and was confirmed to be related to programmed cell death, CHD pathogenesis, and lipid metabolism in the literature. We expected this method could analyze CHD better and more comprehensively from the point of programmed cell death in subnetworks.

\section{Introduction}

Coronary heart disease (CHD) is the narrowing or blockage of the coronary arteries, usually caused by atherosclerosis. Atherosclerosis is the buildup of plaques on the inner walls of the arteries, which can restrict blood flow to the heart muscle by physically clogging the artery or by causing abnormal artery tone and function [1]. In general, apoptosis occurring in atherosclerotic lesions has been suggested to be involved in the evolution and the structural stability of the plaques [2]. Because late apoptotic cells can launch proatherogenic inflammatory responses, adequate engulfment of apoptotic cells (efferocytosis) by macrophages is important to withstand the atherosclerosis progression [3]. Macrophages represent more than $40 \%$ of dead cells in the atherosclerotic lesions [4]. Enhancing intracellular lipid content can enlarge foam cells formation [5]. Formation of lipid-laden foam cells from macrophages and, to a less extent, from smooth muscle cells represents a landmark for atherosclerosis [6]. Some studies have documented that deregulation of apoptosis, a form of genetically programmed cell death, occurs in atherosclerotic lesions $[7,8]$.

Programmed cell death (PCD) is proposed to be death of a cell in pathological format, mediated by an intracellular program [9]. PCD referred to apoptosis, autophagy, and programmed necrosis. These three forms of PCD may jointly decide the fate of cells; apoptosis and programmed necrosis invariably contribute to cell death, whereas autophagy can play either prosurvival or prodeath roles [10]. Imbalance between cell survival and death may contribute to dramatic alterations in cellularity of the arterial wall with atherosclerosis. Carotid Atherosclerosis Progression Trial Investigating Vascular Cholesterol Acyltransferase (ACAT) Inhibition Treatment Effects was developed to assist in the prevention of cardiovascular disease [11]. Cyclooxygenase-2 (COX-2) in control of cell proliferation, apoptosis, played a significant role in the development of atherosclerosis [12,13]. Matrix metalloproteinase produced by macrophages played 
an important role in modulating plaque stability and apoptosis of cell $[14,15]$. Macrophages play a crucial role at all stages of $\mathrm{CHD}$, including regulation of foam cell formation, the inflammatory response, and the stability of atherosclerotic plaques. The effect of enzyme in macrophages and foam cells is of great value in the study of CHD from the perspective of programmed cell death.

Traditional experimental research was through single gene or single compound to analyze $\mathrm{CHD}$, and it could not provide the analysis at system level. Along with the rapid development of technology, the study about biological networks is increasingly providing valuable information on biological systems [16-18]. Metabolic network is an important typical biochemical network which consists of enzymes and chemical compounds $[19,20]$. Due to localization of metabolic enzymes, many metabolic processes involve coordinated interactions between different organelles, and one metabolic step may be dependent upon the successful completion of the previous step $[21,22]$. In other words, chemical compounds can be considered as edges mediating between enzymes. A better understanding of human metabolism and its relationship with diseases is an important task in human systems biology studies. In metabolic reactions, there is a cascade relationship between enzymes. The relationship network between enzymes was constructed to select risk enzymes related disease and reveal the pathogenesis. Considering from this aspect, enzyme marker will be searched and be used for disease detection in the near future.

Therefore, in our paper, we presented a high-quality human metabolic enzyme network, Edinburgh Human Enzyme Network (EHEN), manually reconstructed based on Edinburgh Human Metabolic Network (EHMN) [23], and divide it into different subnetworks, by integrating genome annotation and location information from different databases and metabolic reaction information from the literature. We presented a strategy to select reporter enzyme considering the change of foam cells and macrophages between normal state and disease state with network characteristic. Through the method of selecting modules based reporter enzymes and the functional enrichment analysis, the module related to programmed cell death was analyzed further. We expected this method could analyze CHD better and more comprehensively from the point of programmed cell death in subnetworks.

\section{Materials and Methods}

2.1. Gene Expression Data Source and Data Preprocessing. We used monocyte-derived macrophages from peripheral blood cultured in the absence or presence of oxidized LDL, baseline macrophages or foam cells. The macrophages were obtained from 15 subjects with subclinical atherosclerosis and a family history of CHD. Macrophages from 15 age and gender matched subjects with no atherosclerosis and no family history of $\mathrm{CHD}$ were used as control. We downloaded this expression data with the accession number of GSE9874 [24] from NCBI-GEO database (http://www.ncbi.nlm.nih.gov/geo/).
2.2. Construction of Enzyme Network and Enzyme Subnetwork Based on EHMN. The Edinburgh Human Metabolic Network (EHMN) was reconstructed by integrating genome annotation information from different databases and metabolic reaction information from the literature [23]. EHMN contains nearly 3000 metabolic reactions, which were reorganized into 70 human-specific pathways according to their functional relationships $[25,26]$. Based on EHMN metabolic network, we built an enzyme network which was constructed by enzyme (nodes) and reactions (edges). According to reaction relationships between substrates and products (i.e., the product of a reaction is just right the substrate of the next reaction), corresponding enzymes of reactions were connected to a network, as shown in Figure 1(a). Because the enzyme network construction is based on EHMN, the Edinburgh Human Metabolic Network, we called it the Edinburgh Human Enzyme Network (EHEN).

The enzyme protein location information in this work is extracted mainly from gene ontology (GO) [27]. Files containing GO association in human and the hierarchically organized GO terms ("OBO" file) can be easily downloaded from the GO website. This file is maintained by the GOA group at EBI which aims to provide high-quality GO annotations to proteins in the UniProtKB, as demonstrated in Figure 1(b). The proteins with uncertain locations or other locations not included in the known specific locations were classified to an "uncertain" location, and they will be finally screened out from our study.

\subsection{Selection of Modules Related to Programmed Cell Death} Based on Reporter Enzymes. We proposed a computational approach to select modules related to programmed cell death. All the disease and matched control samples were normalized simultaneously using the robust multiarray average (RMA) method [28], implemented in R/Bioconductor project. We calculated and obtained risk enzymes from the above expression profile of foam cells and macrophages, separately. Next, the common enzymes of two cells were sought as reporter enzymes. Further, the network functional modules related to programmed cell death based on reporter enzymes were selected. Details of our selection progress are as follows.

(1) $P$ values of genes, $P_{-}$value $_{g}$, indicating the significance of differential expression, were converted to standard $Z$-scores $Z_{-}$value $g$ with a mean of 0 and a variance of 1 by using the inverse normal cumulative distribution function $(\mathrm{CDF})\left(\theta^{-1}\right)$ :

$$
Z_{\text {_value }}=\operatorname{cdf}^{-1}\left(1-P_{-} \text {value }_{g}\right) \text {. }
$$

(2) $Z$-scores of enzymes calculated as median of $Z$-scores of the $k$ genes were as follows:

$$
Z_{-} \text {value }_{\text {Enzyme }}=\frac{1}{k} \sum_{i=1}^{n}\left\{Z_{-} \text {value }_{g}\right\} \text {. }
$$

(3) $Z_{\text {_value }}$ Enzyme scores were then corrected for the background distribution by subtracting the mean $\left(\mu_{k}\right)$ 

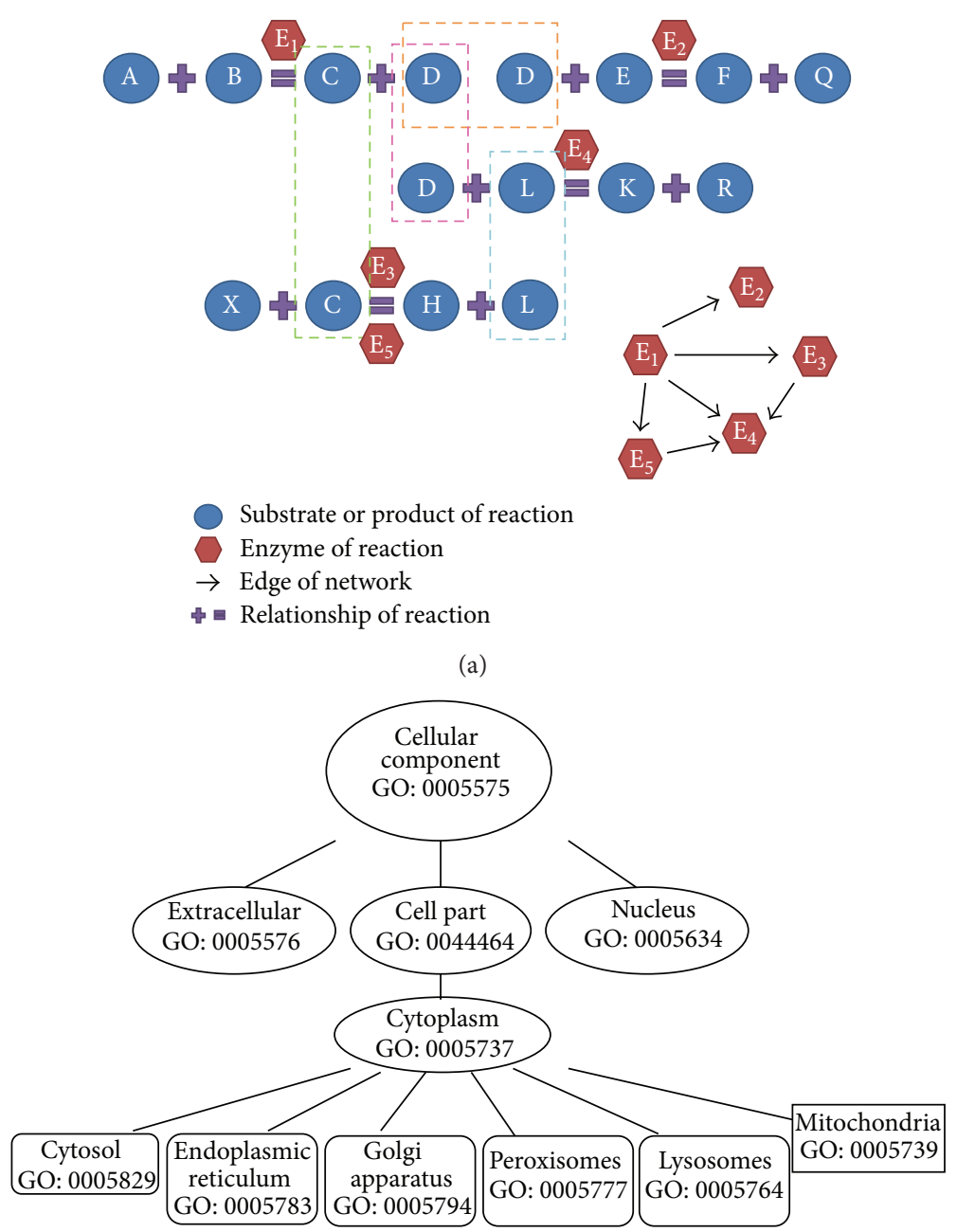

(b)

FIGURE 1: Schematic diagram of enzyme network construction and protein location method. (a) Substrates and products of reactions are shown as blue circles, enzymes of reactions are shown as red hexagons, relationships of reaction are shown as purple mathematical symbols, edges of network are shown as black arrows, and the arrow pointing means the order of reactions. (b) The Gene ontology terms used for protein locations. The top locations are circled. Proteins are matched to GO terms and then backtracked to the selected locations through the hierarchical structure.

and dividing by the standard deviation $\left(\sigma_{k}\right)$ of the aggregated $Z$-scores derived by sampling 10000 sets of $k$ enzymes from the EHEN:

$$
Z_{\text {Enzyme }}^{\text {corrected }}=\frac{Z_{\text {Enzyme }}-\mu_{k}}{\sigma_{k}} .
$$

(4) Corrected $Z$-scores were then transformed to $P$ values by using CDF:

$$
P_{-} \text {value }_{\text {Enzyme }}=1-\operatorname{cdf}\left(Z_{\text {Enzyme }}^{\text {corrected }}\right) .
$$

(5) Reporter enzymes of the EHEN were screened with $P$ values under a significant threshold of 0.05 .

(6) The network functional modules based reporter enzymes were found by MCODE [29] (with the parameters of degree cutoff $\geq 2$ and $K$-core $\geq 2$ ).
(7) Gene ontology (GO) enrichment analysis was performed for each module from 6; select the modules related to programmed cell death.

(8) To repeat the above method in the 6 subnetworks, we got the reporter enzymes related network functional modules based on different subnetworks. Finally, we got reporter enzymes related network functional modules in the EHEN and six subnetworks.

2.4. Functional Programmed Cell Death-Module Analysis of the EHEN and Subnetworks. In order to analyze the relationship between cell death and $\mathrm{CHD}$, we select the functional modules related to programmed cell death for further research. GO functional enrichment analysis was applied for these modules using the Functional 
Annotation Tool in DAVID Bioinformatics Resources 6.7 (http://david.abcc.ncifcrf.gov/). FDR less than 0.05 was considered as significant.

\section{Result}

3.1. Enzyme Network and Subnetwork from the EHMN. Using the method described above in the network and subnetwork construction, all the human proteins coming from GO were classified into the chosen locations. By the described method, our constructed EHEN was separated into 6 subnetworks according to its localization information in our study, including cytoplasm (EHEN-C), mitochondria (EHEN-M), Golgi apparatus (EHEN-G), extracellular (EHEN-E), nucleus (EHEN-N), and endoplasmic reticulum (EHEN-R).

The input for network topological analysis to the calculation is the list of relationships of enzymes. Observing the degree distribution, characteristic path length, the connectivity, network diameter, the average and the maximum of the shortest path lengths, and clustering coefficient, the EHEN and subnetworks were found as scale-free following nearly a power law model $\left(f(x)=a * x^{b}, a=29.9\right.$, and $b=-0.46)$ and had small-world properties with scalefree topology, which was a general characteristic of complex biological networks (more topological characteristics of networks are in the Supplementary Material Table S1 and Figure S1 (see Supplementary Material available online at http://dx.doi.org/10.1155/2014/475379)).

\subsection{The Modules Based Reporter Enzymes in EHEN and Sub-} networks. From the reporter enzyme selection algorithms in the method part, we got reporter enzymes of overall and subnetwork, respectively. The numbers of reporter enzymes varied in different networks; the EHEN-C had the largest number of reporter enzymes, while the EHEN-R had only 2 selected reporter enzymes; the overall EHEN network stayed in the middle position (7 reporter enzymes), as listed in the Supplementary Material (Table S2: the reporter enzymes of overall and subnetwork). The degree and the clustering coefficient of the reporter enzymes had significant difference $(P<0.05)$ in their respective networks. It was shown that the reporter enzymes had smaller degree and clustering coefficient in the EHEN, while they had bigger degree and clustering coefficient in subnetworks (more topological characteristics of reporter enzymes are in the Supplementary Material Table S3). It implied that the reporter enzymes selected from subnetworks played a more important part in the disease emergence and tended to form network modules.

With the help of Cytoscape and its plug-ins, we selected out modules based reporter enzymes. From module division and annotation result of the EHEN and subnetworks by using Mcode, we gained functional modules of each network. Gene ontology (GO) enrichment analysis was performed for seven modules using DAVID, including 2 modules in the EHEN, 2 modules in the EHEN-C, and 3 modules in the EHEN$M$ (see Figure 2, and more information of modules in the Supplementary Material Table S4).
3.3. Literature Retrieval and Functional Annotation of the Reporter Enzyme and Related Modules. CHD was caused by the plaques buildup of lipid, which was created by macrophages forming foam cells. We defined the classification principles based on CHD-related functions-CHD pathogenesis, lipid metabolism, and programmed cell death (Table 1). Most of the modules could be enriched to the basic metabolic function class, the related CHD pathogenesis, and the related lipid function class. For instance, asparagine synthase was a reporter enzyme in both the EHEN and the EHEN-M and significantly inhibited the proliferation of cells [30]. In the module EHEN-M2, another reporter enzyme ec: 1.14.15.6, cholesterol monooxygenase (CYP11A1), also known as cytochrome P-450scc was the most important family of enzymes in microsomal mixed function oxidase, widely distributed in vivo, and it was detected human cardiac expression of the mRNAs for many of the enzymes involved in the formation of adrenal corticosteroids, supporting the possibility of local production of corticosteroids and a physiological role for these hormones in cardiac function [31]. In the module EHEN-C1, the reporter enzyme ec: 6.1.1.3, threonyl-tRNA synthetase (TARS), was an autoantigen in the autoimmune disorder myositis, and borrelidin, which was a potent inhibitor of TARS, inhibits angiogenesis. TARS thus provided a potential target for detecting or interdicting disease-related inflammatory or angiogenic responses [32]. And the reporter enzyme ec: 3.1.4.37 $\left(2^{\prime}, 3^{\prime}\right.$-cyclic-nucleotide $3^{\prime}$-phosphodiesterase), which was enriched on the function classification related lipid, regulates intracellular cAMP levels, which might represent novel therapeutic agents to limit angiogenesis in complex human diseases [33].

Because coronary heart disease is caused by abnormal lipid metabolism, so the GO terms associated with lipid metabolism can be direct evidence between reporter enzymes and $\mathrm{CHD}$. We were more interested in related programmed cell death reporter enzymes and modules.

3.4. Literature Retrieval and Functional Annotation of the Module Related to Programmed Cell Death. The module 3 in mitochondria subnetwork (EHEN-M3) was a module which enriched to not only the basic metabolic function class, the related CHD pathogenesis, but also the related programmed cell death function class. It included 33 enzymes (2 reporter enzymes ec: 6.1.1.7 and ec: 6.1.1.17 and 31 others) (Figure 3(a)). Through GO enrichment analysis, the genes of these enzymes in the module enriched 47 GO terms, including 15 GO terms related programmed cell death, $14 \mathrm{GO}$ terms related $\mathrm{CHD}$, and 18 GO terms related basic metabolic (Figure 3(b)). In this module, we were interested in the part of programmed cell death. For the reporter enzyme ec: 6.1.1.17 (glutamate-tRNA synthetase), it was proved that glutamate-tRNA synthetase of Bacillus subtilis was known to result in the death of the host cell [34]. For the reporter enzyme ec: 6.1.1.7 (alanyltRNA synthetase), it was proved that a homozygous missense mutation in AARS2 causes perinatal or infantile cardiomyopathy with near-total combined mitochondrial respiratory chain deficiency in the heart, in which AARS2 is identified to encode mitochondrial alanyl-tRNA synthetase [35]. 

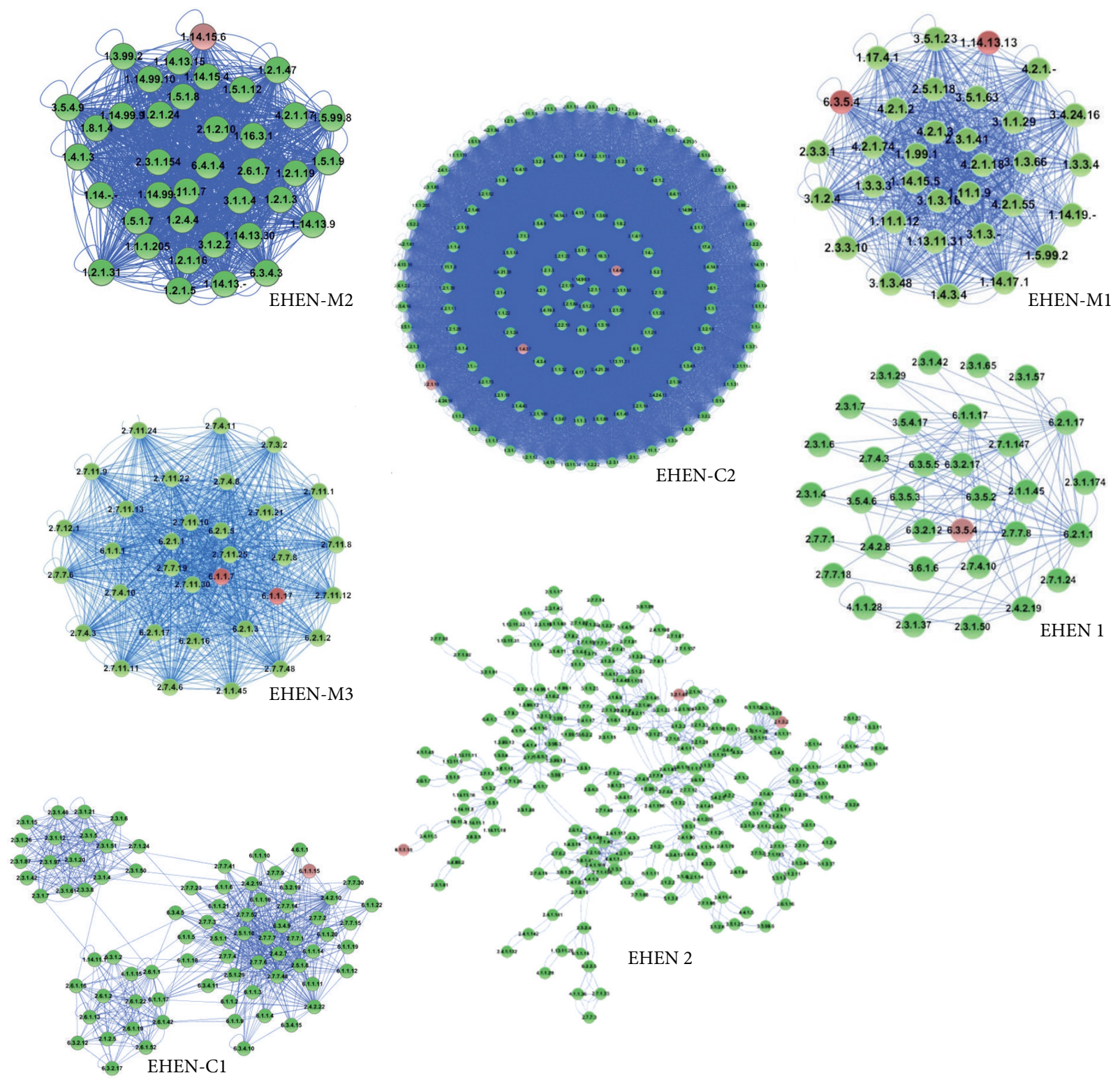

FIGURE 2: Seven modules based on reporter enzymes.

TABLE 1: Module information of overall and part of subnetworks.

\begin{tabular}{lccccc}
\hline \multirow{2}{*}{ Network } & \multirow{2}{*}{ Module count } & \multirow{2}{*}{ RE count } & Basic metabolic & Lelated function count & CHD \\
\hline EHEN & 2 & 4 & 32 & 18 & 9 \\
EHEN-C & 2 & 6 & 40 & 22 & - \\
EHEN-M & 3 & 5 & 51 & 13 & - \\
\hline
\end{tabular}

We found there were 13 enzymes enriched to GO terms related programmed cell death, and more of them belonged to serine/threonine kinases. For instance, the enzyme ec: 2.7.11.10, the inhibitor of $\mathrm{NF}-\kappa \mathrm{B}$ kinase subunit $\beta$ (IKK $\beta)$, formed a transduction complex that controls the production of proinflammatory cytokines mediating cardiomyocyte hypertrophy, and activation of IKK $\beta$ in turn enhances fetal gene expression and cardiomyocyte growth [36]. The enzyme ec: 2.7.11.22 (cyclin-dependent kinase) could interact with p27 in neonatal rat cardiomyocytes, which exerted antiapoptotic and growth-inhibitory effects, and may help to improve heart function and survival in rodents $[37,38]$. 


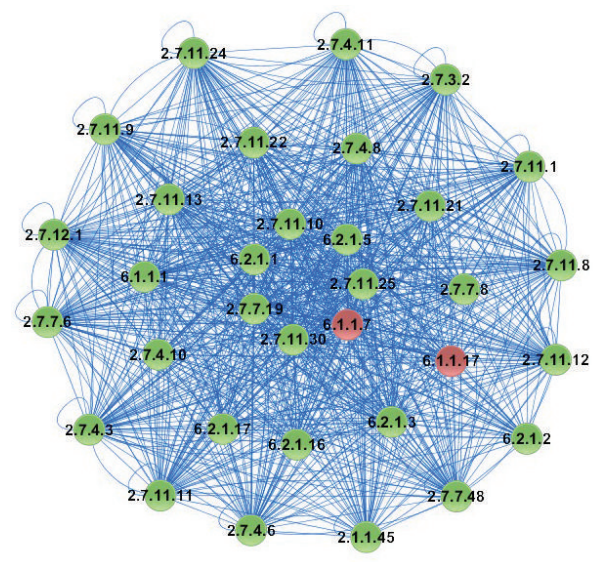

(a)

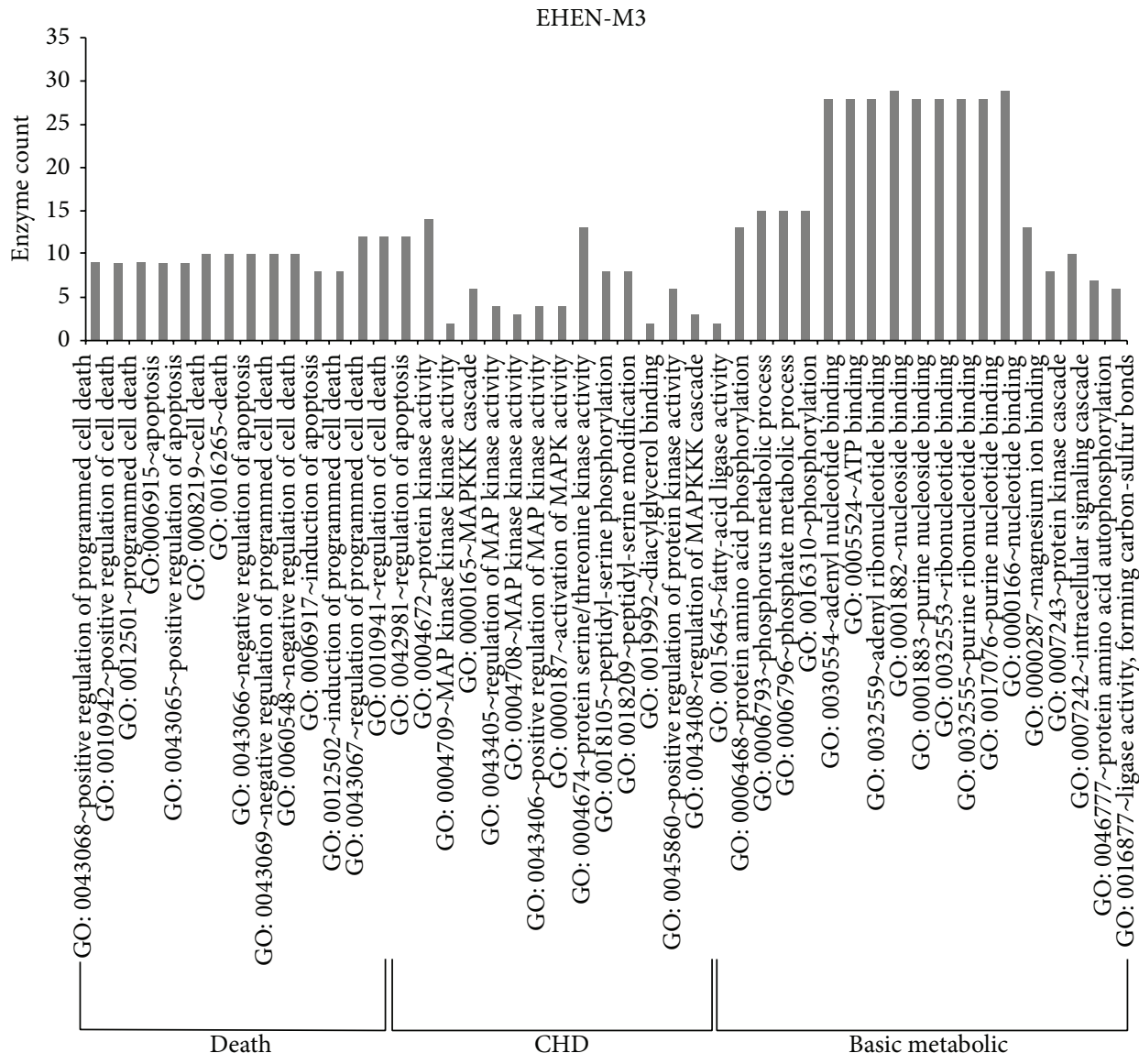

(b)

Figure 3: The module EHEN-M3 and the result of the functional enrichment. (a) The module 3 in EHEN-M. (b) The result of the functional enrichment.

\section{Discussion}

Traditionally, there are several ways to research the mechanism of CHD. Our approach focused on CHD in the programmed cell death perspective on the systems biology level. We constructed a correlative network of enzymes considering the cascade. The differences from the macrophages to the foam cell between disease patients and normal controls were calculated according to the expression level. We proposed a computational approach to select 7 modules based on reporter enzymes ( 2 in the EHEN, 2 in the EHEN-C, and 3 in the EHEN-M). Most of the modules could be enriched to the basic metabolic function class, the related CHD pathogenesis, and the related lipid function class. 
The module EHEN-M3 was related to programmed cell death in the EHEN-M. Functional and structural integrity of mitochondria was essential for the physiological function of the cardiovascular system. Accumulation of mitochondrial DNA mutations has been linked to ischaemic heart disease, cardiomyopathy, and atherosclerotic vascular disease. Mitochondria are known to regulate apoptotic and autophagic pathways that have been shown to play an important role in the development of cardiomyopathy and atherosclerosis [39]. Our results also verified it from a new perspective of enzyme, which was a new research direction about CHD.

It was reported that macrophages and foam cells were associated with CHD in the aspect of programmed cell death. Wang et al. reported that macrophage with lipid growing eventually formed foam cells until death. A large pool of bubble formation and programmed cell death eventually developed into a typical plaque [40, 41]. More importantly, the reporter enzymes were obtained from macrophages and foam cells, which might be isolated from the peripheral blood of patient. Modules could be identified based on these reporter enzymes to reflect the disease state.

Deficiency or alterations in metabolic functions were known to be involved in CHD. Enzyme proteins and chemical compounds were connected in metabolic networks. The reconstruction of the enzyme network could illustrate cascade relationships of enzymes. The selected reporter enzymes and modules from each subnetwork were more closely associated with CHD. We hope that the research would be more comprehensive with more data accumulation, such as posttranslational regulatory data. Considering from this aspect, enzyme marker and modules could be searched and be used in disease detection in the future.

\section{Conflict of Interests}

The authors declare that there is no conflict of interests regarding the publication of this paper.

\section{Authors' Contribution}

$\mathrm{Xu}$ Jia, Wan Li, and Zhengqiang Miao contributed equally to this work.

\section{Acknowledgments}

This work was supported in part by the National Natural Science Foundation of China (Grant no. 61272388), the Natural Science Foundation of Heilongjiang Province (Grant no. F201237), the Science \& Technology Research Project of the Heilongjiang Ministry of Education (Grant no. 12511271), and the Master Innovation Funds of Heilongjiang Province (Grant nos. YJSCX2012-209HLJ and YJSCX2012-224HLJ).

\section{References}

[1] M. J. A. Williams, N. J. Restieaux, and C. J. S. Low, "Myocardial infarction in young people with normal coronary arteries," Heart, vol. 79, no. 2, pp. 191-194, 1998.
[2] C. Soldani, A. I. Scovassi, U. Canosi, E. Bramucci, D. Ardissino, and E. Arbustini, "Multicolor fluorescence technique to detect apoptotic cells in advanced coronary atherosclerotic plaques," European Journal of Histochemistry, vol. 49, no. 1, pp. 47-52, 2005.

[3] E. A. van Vré, H. Ait-Oufella, A. Tedgui, and Z. Mallat, "Apoptotic cell death and efferocytosis in atherosclerosis," Arteriosclerosis, Thrombosis, and Vascular Biology, vol. 32, no. 4, pp. 887893, 2012.

[4] F. D. Kolodgie, J. Narula, A. P. Burke et al., "Localization of apoptotic macrophages at the site of plaque rupture in sudden coronary death," The American Journal of Pathology, vol. 157, no. 4, pp. 1259-1268, 2000.

[5] L. Sanjurjo, N. Amezaga, C. Vilaplana et al., "The scavenger protein apoptosis inhibitor of macrophages (AIM) potentiates the antimicrobial response against mycobacterium tuberculosis by enhancing autophagy," PLoS ONE, vol. 8, p. e79670, 2013.

[6] H. Liao, T. Kodama, and Y. Geng, "Expression of class A scavenger receptor inhibits apoptosis of macrophages triggered by oxidized low density lipoprotein and oxysterol," Arteriosclerosis, Thrombosis, and Vascular Biology, vol. 20, no. 8, pp. 1968-1975, 2000.

[7] Y.-J. Geng and P. Libby, "Evidence for apoptosis in advanced human atheroma: colocalization with interleukin- $\beta$-converting enzyme," The American Journal of Pathology, vol. 147, no. 2, pp. 251-266, 1995.

[8] M. M. Kockx, G. R. Y. De Meyer, J. Muhring, H. Bult, J. Bultinck, and A. G. Herman, "Distribution of cell replication and apoptosis in atherosclerotic plaques of cholesterol-fed rabbits," Atherosclerosis, vol. 120, no. 1-2, pp. 115-124, 1996.

[9] H. Engelberg-Kulka, S. Amitai, I. Kolodkin-Gal, and R. Hazan, "Bacterial programmed cell death and multicellular behavior in bacteria," PLoS Genetics, vol. 2, no. 10, article e135, 2006.

[10] L. Ouyang, Z. Shi, S. Zhao et al., "Programmed cell death pathways in cancer: a review of apoptosis, autophagy and programmed necrosis," Cell Proliferation, vol. 45, no. 6, pp. 487498, 2012.

[11] M. C. Meuwese, E. de Groot, R. Duivenvoorden et al., "ACAT inhibition and progression of carotid atherosclerosis in patients with familial hypercholesterolemia the CAPTIVATE randomized trial," JAMA, vol. 301, no. 11, pp. 1131-1139, 2009.

[12] C. Cahlin, C. Lönnroth, A. Arvidsson, S. Nordgren, and K. Lundholm, "Growth associated proteins in tumor cells and stroma related to disease progression of colon cancer accounting for tumor tissue PGE2 content," International Journal of Oncology, vol. 32, no. 4, pp. 909-918, 2008.

[13] M. Sanak, H. Plutecka, W. Szczeklik, W. Piwowarska, P. Rostoff, and A. Szczeklik, "Functional promoter polymorphism of cyclooxygenase- 2 modulates the inflammatory response in stable coronary heart disease," Polskie Archiwum Medycyny Wewnetrznej, vol. 120, no. 3, pp. 82-88, 2010.

[14] L. J. Zhang, W. X. Liu, Y. D. Chen, X. T. Song, Z. N. Jin, and S. Lü, "Proliferation, migration and apoptosis activities of endothelial progenitor cells in acute coronary syndrome," Chinese Medical Journal, vol. 123, no. 19, pp. 2655-2661, 2010.

[15] T. G. Ashlin, A. P. Kwan, and D. P. Ramji, "Regulation of ADAMTS-1, -4 and -5 expression in human macrophages: differential regulation by key cytokines implicated in atherosclerosis and novel synergism between TL1A and IL-17," Cytokine, vol. 64, pp. 234-242, 2013. 
[16] A. J. Enright, I. Illopoulos, N. C. Kyrpides, and C. A. Ouzounis, "Protein interaction maps for complete genomes based on gene fusion events," Nature, vol. 402, no. 6757, pp. 86-90, 1999.

[17] S. Fields, "The future is function," Nature Genetics, vol. 15, no. 4, pp. 325-327, 1997.

[18] J. C. Rain, L. Selig, H. de Reuse et al., "The protein-protein interaction map of Helicobacter pylori," Nature, vol. 409, no. 6817, pp. 211-215, 2001.

[19] A. Zelezniak, T. H. Pers, S. Soares, M. E. Patti, and K. R. Patil, "Metabolic network topology reveals transcriptional regulatory signatures of type 2 diabetes," PLoS Computational Biology, vol. 6, no. 4, Article ID e1000729, 2010.

[20] M. G. Heiden Vander, S. Y. Lunt, T. L. Dayton et al., "Metabolic pathway alterations that support: cell proliferation," Cold Spring Harbor Symposia on Quantitative Biology, vol. 76, pp. 325-334, 2011.

[21] N. B. Larson, G. D. Jenkins, M. C. Larson et al., "Kernel canonical correlation analysis for assessing gene-gene interactions and application to ovarian cancer," European Journal of Human Genetics, vol. 22, pp. 126-131, 2014.

[22] H. Chen and V. Vanburen, "A review of integration strategies to support gene regulatory network construction," The Scientific World Journal, vol. 2012, Article ID 435257, 12 pages, 2012.

[23] H. Ma, A. Sorokin, A. Mazein et al., "The Edinburgh human metabolic network reconstruction and its functional analysis," Molecular Systems Biology, vol. 3, article 135, 2007.

[24] D. Steinberg, "Low density lipoprotein oxidation and its pathobiological significance," The Journal of Biological Chemistry, vol. 272, no. 34, pp. 20963-20966, 1997.

[25] D. Cotter, A. Maer, C. Guda, B. Saunders, and S. Subramaniam, "LMPD: LIPID MAPS proteome database," Nucleic Acids Research, vol. 34, pp. D507-D510, 2006.

[26] N. C. Duarte, S. A. Becker, N. Jamshidi et al., "Global reconstruction of the human metabolic network based on genomic and bibliomic data," Proceedings of the National Academy of Sciences of the United States of America, vol. 104, no. 6, pp. 17771782, 2007.

[27] M. Ashburner, C. A. Ball, J. A. Blake et al., "Gene ontology: tool for the unification of biology," Nature Genetics, vol. 25, no. 1, pp. 25-29, 2000.

[28] R. A. Irizarry, Z. Wu, and H. A. Jaffee, "Comparison of Affymetrix GeneChip expression measures," Bioinformatics, vol. 22, no. 7, pp. 789-794, 2006.

[29] G. D. Bader and C. W. V. Hogue, "An automated method for finding molecular complexes in large protein interaction networks," BMC Bioinformatics, vol. 4, no. 1, p. 2, 2003.

[30] B. Zhang, L.-W. Dong, Y.-X. Tan et al., "Asparagine synthetase is an independent predictor of surgical survival and a potential therapeutic target in hepatocellular carcinoma," British Journal of Cancer, vol. 109, no. 1, pp. 14-23, 2013.

[31] K. M. Kayes-Wandover and P. C. White, "Steroidogenic enzyme gene expression in the human heart," Journal of Clinical Endocrinology and Metabolism, vol. 85, no. 7, pp. 2519-2525, 2000.

[32] T. F. Williams, A. C. Mirando, B. Wilkinson, C. S. Francklyn, and K. M. Lounsbury, "Secreted Threonyl-tRNA synthetase stimulates endothelial cell migration and angiogenesis," Scientific Reports, vol. 3, article 1317, 2013.

[33] S. J. Netherton and D. H. Maurice, "Vascular endothelial cell cyclic nucleotide phosphodiesterases and regulated cell migration: implications in angiogenesis," Molecular Pharmacology, vol. 67, no. 1, pp. 263-272, 2005.
[34] J. W. Baick, J. H. Yoon, S. Namgoong et al., "Growth inhibition of Escherichia coli during Heterologous expression of Bacillus subtilis glutamyl-tRNA synthetase that catalyzes the formation of mischarged glutamyl-tRNA1Gln," The Journal of Microbiology, vol. 42, no. 2, pp. 111-116, 2004.

[35] A. Götz, H. Tyynismaa, L. Euro et al., "Exome sequencing identifies mitochondrial alanyl-tRNA synthetase mutations in infantile mitochondrial cardiomyopathy," American Journal of Human Genetics, vol. 88, no. 5, pp. 635-642, 2011.

[36] C. D. del Vescovo, S. Cotecchia, and D. Diviani, "A-kinaseanchoring Protein-Lbc anchors $\mathrm{I} \kappa \mathrm{B}$ kinase $\beta$ to support interleukin-6-mediated cardiomyocyte hypertrophy," Molecular and Cellular Biology, vol. 33, no. 1, pp. 14-27, 2013.

[37] F. Konecny, J. Zou, M. Husain, and R. von Harsdorf, "Postmyocardial infarct p27 fusion protein intravenous delivery averts adverse remodelling and improves heart function and survival in rodents," Cardiovascular Research, vol. 94, no. 3, pp. 492-500, 2012.

[38] A. H. Schönthal, "Measuring cyclin-dependent kinase activity." Methods in Molecular Biology, vol. 281, pp. 105-124, 2004.

[39] E. A. Dominic, A. Ramezani, S. D. Anker, M. Verma, N. Mehta, and M. Rao, "Mitochondrial cytopathies and cardiovascular disease," Heart, vol. 100, no. 8, pp. 611-618, 2014.

[40] J. C. Wang and M. Bennett, "Aging and atherosclerosis: mechanisms, functional consequences, and potential therapeutics for cellular senescence," Circulation Research, vol. 111, no. 2, pp. 245-259, 2012.

[41] A. Ghattas, H. R. Griffiths, A. Devitt, G. Y. Lip, and E. Shantsila, "Monocytes in coronary artery disease and atherosclerosis: where are we now?" Journal of the American College of Cardiology, vol. 62, pp. 1541-1551, 2013. 


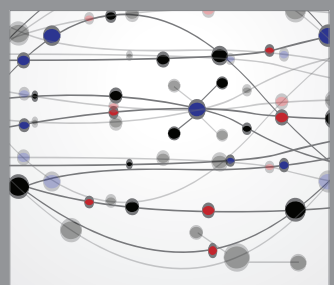

The Scientific World Journal
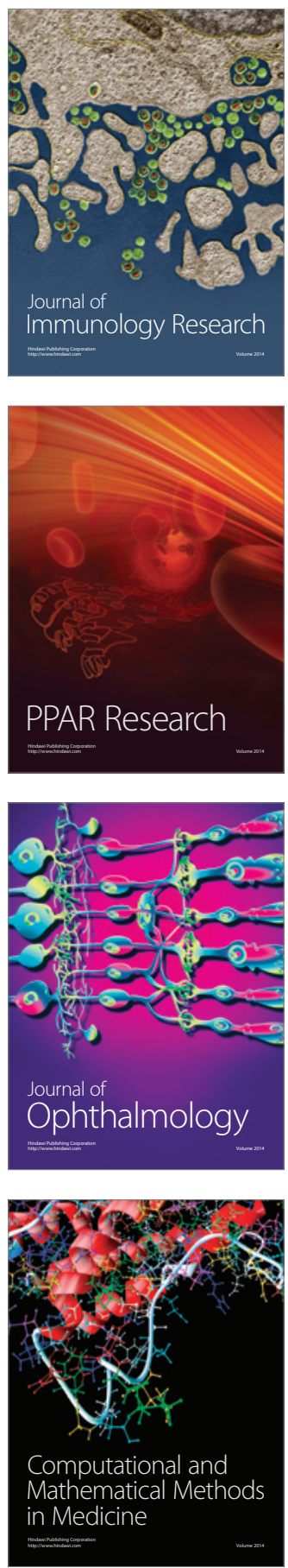

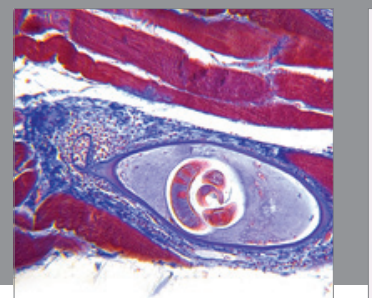

Gastroenterology

Research and Practice
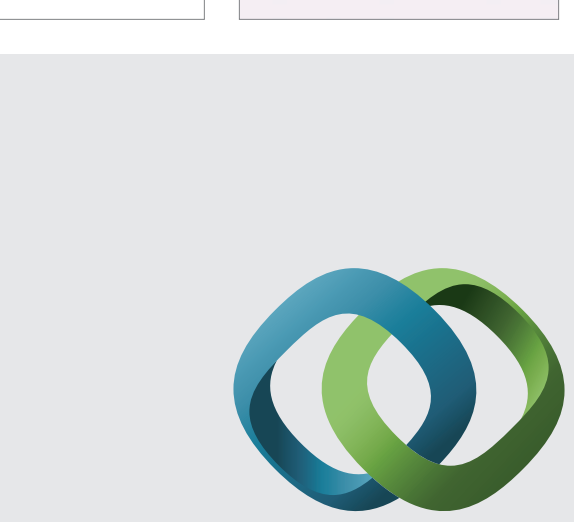

\section{Hindawi}

Submit your manuscripts at

http://www.hindawi.com
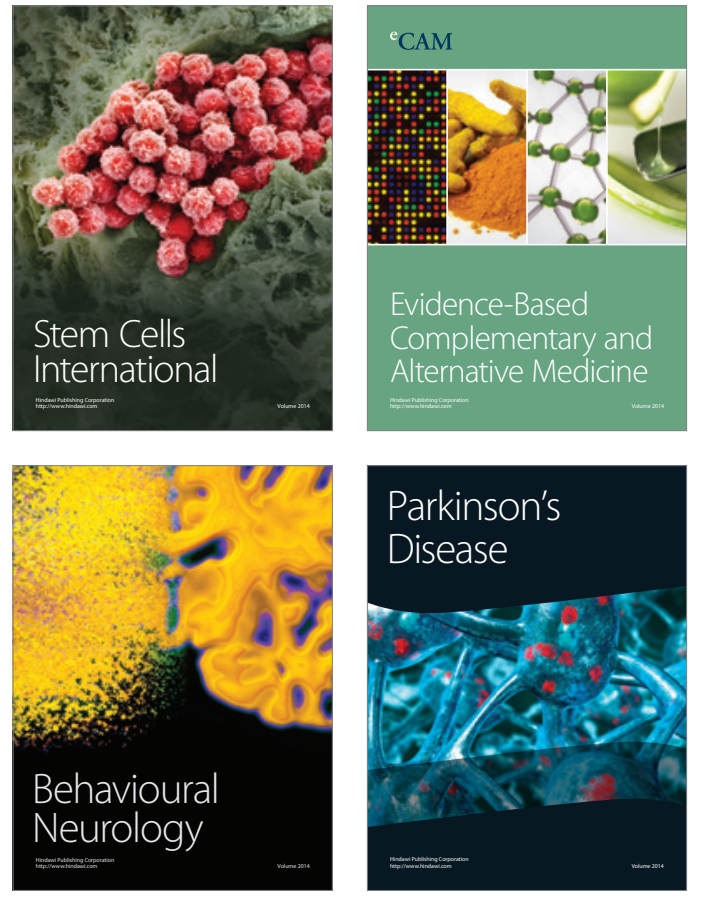
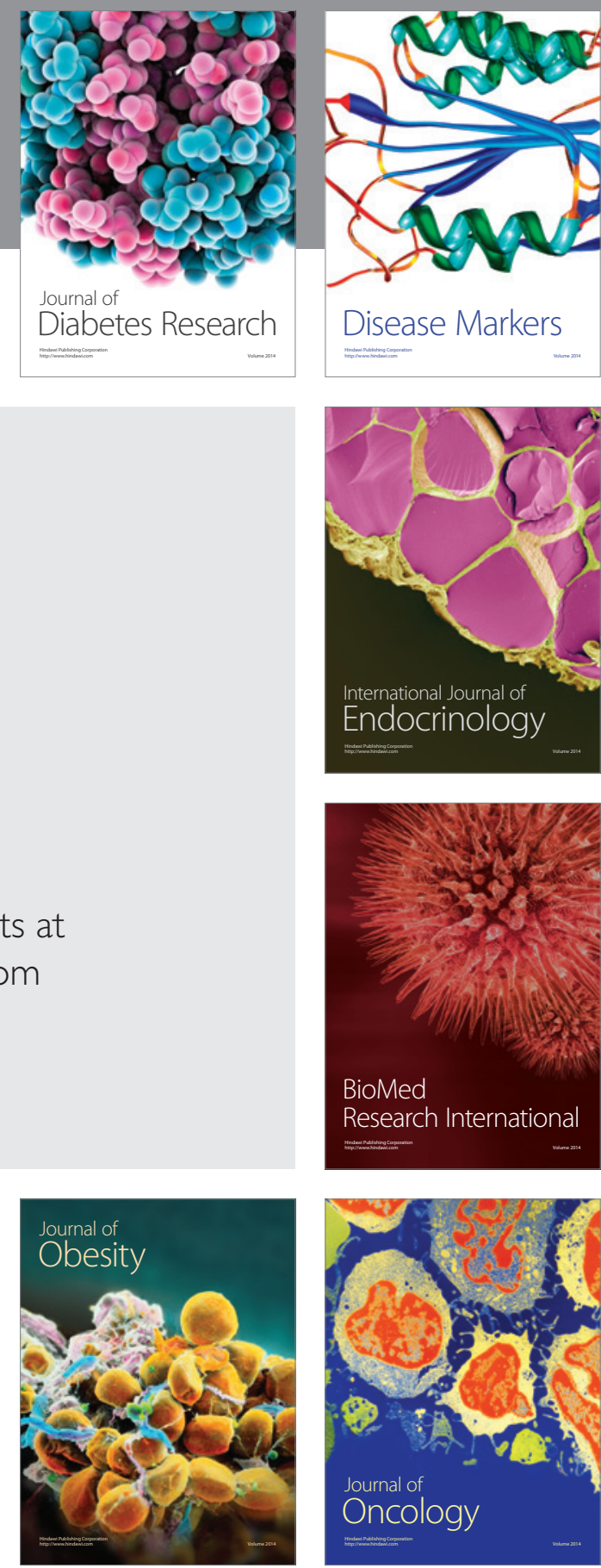

Disease Markers
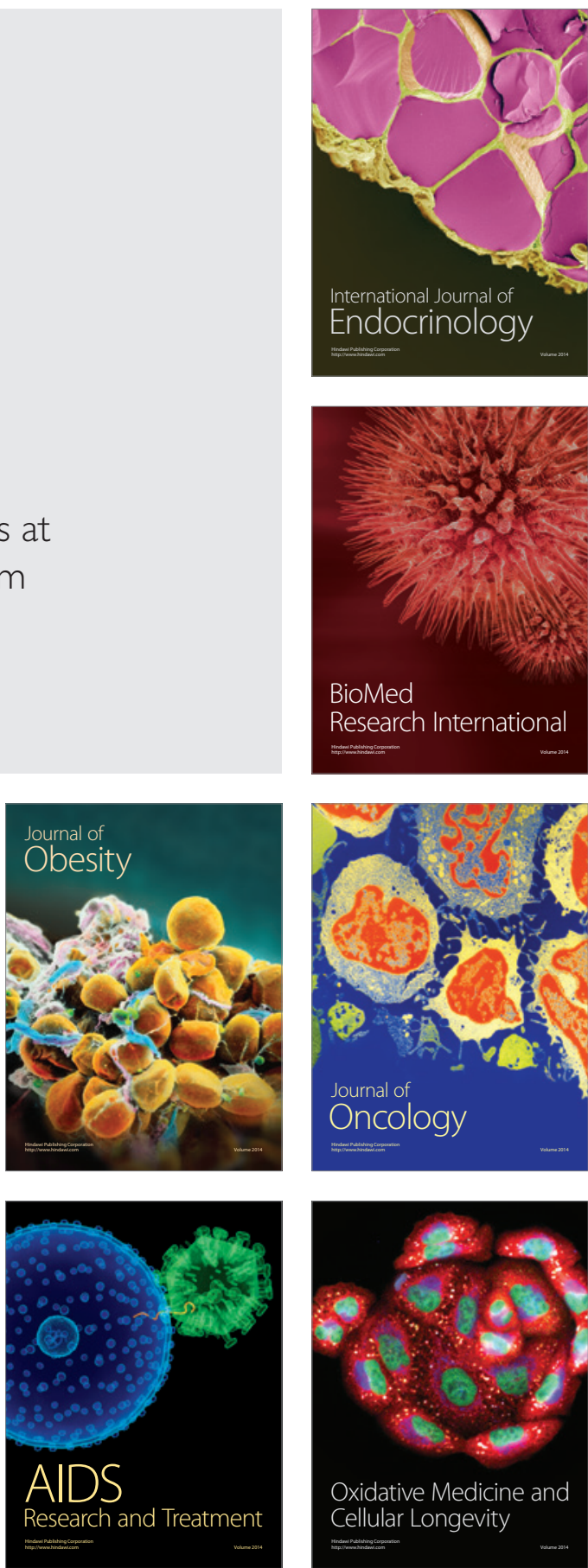